\section{Schon ein paar Tage Faulheit schwächen die Glukoseroleranz}

\author{
Die akute Reduktion körperlicher Aktivität beeinflusst Insulinsensitivität \\ und Glukosestoffwechsel negativ - sehr schnell und recht nachhaltig.
}

Hier steht eine Anzeige.

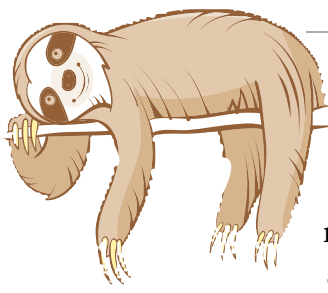

- Wenn wir uns fragen, wie viele Stunden am Tag wir uns eigentlich aktiv bewegen, erleben wir eine Überraschung: Der Durchschnittsdeutsche ist 23

Stunden und 40 Minuten lang inaktiv! In den verbleibenden 20 Minuten schaffen wir es, ungefähr 2.500 Schritte zurückzulegen - das ist der Grad körperlicher Aktivität, den wir im Schnitt realisieren.

In den letzten drei Jahren wurde in vielen Studien der Zusammenhang von metabolischen und kardiovaskulären Risikofaktoren mit körperlicher Alltagsaktivität untersucht. Hierbei zeigte sich, dass Alltagsaktivität die Glukosestoffwechsellage fast immer bessert. Daraus leiten wir evidenzbasiert die Empfehlungen für mindestens 5.000 Schritte pro Tag und das Gesundheitsziel von 10.000 Schritten ab. Wenig erforscht ist bisher die Frage, was passiert, wenn Menschen mit einem relativ hohen Aktivitätslevel plötzlich inaktiv werden.

Für eine Studie in Missouri/USA wurden 14 gesunde, aktive, $24 \pm 1,1$ Jahre alte Männer untersucht. Sie erhielten einen oralen Glukosetoleranztest am Beginn der Untersuchung, nach fünf Tagen "Faulheit" mit $<5.000$ Schritten am Tag und nach einem weiteren Tag mit Aktivität $>10.000$ Schritten. Die Insulinsensitivität wurde bestimmt und die Nüchtern- und postprandiale Glukoseexklusion mit kontinuierlichem Monitoring analysiert. Zudem wurde der Blutfluss in der Arteria brachialis und femoralis untersucht.

Die Veränderung der körperlichen Aktivität hatte keinen Einfluss auf den Blutfluss in den genannten Gefäßen. Allerdings führten fünf Tage Inaktivität zu einem signifikanten Abfall der Insulin- sensitivität $(11,3 \pm 1,5 \mathrm{zu} 8,0 \pm 1,0 ; \mathrm{p}<$ $0,05)$. Den gleichen Effekt konnte man bei der postprandialen Glukose beobachten, die von $113 \pm 3$ auf $123 \pm 5 \mathrm{mg} /$ $\mathrm{dl}$ stieg $(\mathrm{p}<0,05)$. Interessanterweise konnten die Insulinsensitivität und der Glukosemetabolismus nach einem weiteren Tag gesteigerter körperlicher Aktivität nicht wieder normalisiert werden.

- Reynolds LJ, Credeur DP, Holwerda SW et al. Acute inactivity impairs glycemic control but not blood flow to glucose ingestion. Med Sci Sports Exerc. 2015;47:1087-94

\section{KOMMENTAR}

Man muss regelmäßig körperlich aktiv sein. Unseren Patienten und auch uns selbst erzählen wir das immer wieder-aber kaum einer setzt dieses Wissen im Alltag um. Die Studie zeigt in einer kleinen Klientel von gesunden, aktiven Männern, dass plötzliche Inaktivität die Insulinsensitivität sehr schnell nachteilig beeinflusst und dass die kurzfristige Rückkehr zu mehr körperlicher Aktivität das nicht genauso schnell wieder normalisieren kann. Den Unterschied von $10 \mathrm{mg} / \mathrm{dl}$ Glukose kennen wir bereits: Er ist in etwa identisch mit jenem, der sich - mit umgekehrtem Vorzeichen - in großen prospektiven Präventionsstudien zeigt.

Die Ergebnisse sind besonders wichtig, wenn wir uns überlegen, wie schnell unser Alltag dazu führen kann, dass wir unser Aktivitätslevel reduzieren - man denke an berufliche Veränderungen, aber auch an den Technologiefortschritt mit Smart Health, Smart Business und Mobile Solutions. Viele Neuerungen helfen uns im Alltag sehr, führen aber oft auch zu mehr Bequemlichkeit. Kurzfristige Änderungen im Aktivitätslevel (Stichwort: einmal wöchentlich ins Fitnessstudio) können diesen Effekt nicht aufhalten. Diese Ergebnisse sind erschreckend klar und enden in der Quintessenz: Nur kontinuierliche Aktivität eines bestimmten Levels hilft, dem Diabetes mellitus davonzulaufen.

Prof. Dr. med. P. Schwarz 